\title{
$A$ Note on Citations
}

Wagnerian passages are cited either by text cues ("Hagen, was tatest du?"), or, where greater precision is demanded, by measure numbers or reference to the Schirmer vocal scores (using page/system/measure within system: 50/3/1-4). This method of citation allows readers to find their way without undue difficulty in any one of the many full-score editions rather than requiring them to use one particular edition.

The Verdi scores available present, if anything, an even more chaotic situation than that for Wagner. The articles on Ernani and Rigoletto, which grew out of work on the Complete Verdi edition, make direct reference to the relevant volumes of that edition. The remainder of the Verdi citations refer to the standard Ricordi vocal scores (again using page/system/measure within system) unless the nature of the discussion requires another method.

Needless to say, reference to vocal scores is solely for the convenience of readers and is not meant to suggest that consultation of the full orchestral scores is anything less than indispensable for those who wish to analyze this repertoire. 
